\title{
Using machine learning algorithm for diagnosis of stomach disorders
}

\author{
Amirgaliyev Y. $1,2[0000-0002-6528-0619]$, Shamiluulu Sh. ${ }^{1,2[0000-0003-1627-704 X]}$, \\ Merembayev T. ${ }^{1,3[0000-0001-8185-235 X]}$, and Yedilkhan D. ${ }^{[0000-0002-6528-0619]}$ \\ ${ }^{1}$ Institute Information and Computational Technologies CS MES RK, Almaty, \\ Kazakhstan \\ amir_ed@mail.ru \\ 2 Suleyman Demirel University, Kaskelen, Kazakhstan \\ shahriar.shamiluulu@sdu.edu.kz \\ 3 International Information Technology University, Almaty, Kazakhstan \\ timur.merembayev@gmail.com
}

\begin{abstract}
Medicine is one of the rich source of data, generating and storing massive data, begin from description of clinical symptoms and end by different types of biochemical data and images from devices. Manual search and detecting biomedical patterns is complicate task from massive data. Data mining can improve the process of detecting patterns. Stomach disorders are most common disorders that affect over $60 \%$ of human population. In this work, the classification performance of four non linear supervised learning algorithms i.e. Logit, K-Nearest Neighbour, XGBoost and LightGBM for five types of stomach disorders are compared and discussed. The objectives of this research is to find trends of using or improvements of machine learning algorithms for detecting symptoms of stomach disorders, to research problems of using machine learning algorithms for detecting stomach disorders. Bayesian optimization is considered to find optimal hyper parameters in the algorithms, which is faster than the grid search method. Results of the research shows algorithms that base on gradient boosting technique (XGBoost and LightGBM) get better accuracy more $95 \%$ on test dataset. For diagnostic and confirmation of diseases need to improve accuracy, in the article we propose to use optimization methods for accuracy improvement with using machine learning algorithms.
\end{abstract}

Keywords: Stomach disorder · machine learning algorithm · decision support system · Bayesian optimization.

\section{Introduction}

Computer applications and tools are being used in almost every field to assist the work on a daily basis and the medicine is not an exception to that [1]. Currently there are various Machine Learning (ML) methods that are being applied for disease diagnosis. It is strongly believed that it will be more widely used in biomedical systems in this century [2]. This is because of high complexity 
included in the clinical data. The aim of the work is, to perform a comparative study between four ML methods on stomach disorders and identify the top ones according to performance metrics which can be incorporated into clinical decision support system. Gastrointestinal diseases are considered to be one of the most common disorders which affect more than $46 \%$ of human population, where over $60 \%$ of the population is affected by the stomach disorders [3]. Generally, it is easier to find a medical data related to digestive system disorders and ethical reports are more likely to be accepted and permission is more likely to be granted to conduct research. Due to the aforementioned reasons, five stomach diseases were selected: stomach flu, gastroesophageal reflux (heartburn), gastritis, peptic ulcer, and stomach cancer [4]. Currently, there is a continuous on-going research in the field of medical diagnosis and treatment. A tremendous work has been done by applying the supervised learning methods i.e., Neural Networks, Regression models, and Support Vector Machines for diagnosis diseases like diabetes, heart attack, cancer, and kidney diseases $[2,5,6]$. Further the methods background information and implications in medical field is described.

\section{Background information and implications}

This sections provides brief information on four ML algorithms i.e., Logistic Regression (Logit), K-Nearest Neighbour, XGBoost and LightGBM. Two last algorithms are similar and built under gradient boosting method. LightGBM differs from XGBoost by specific features, especially process of creation trees. Parameters for tuning model are almost similar for both algorithms. Each section also describes the implications of algorithms and provides plausible outcomes.

\subsection{Logistic regression model}

In statistics, the Logit has a wide range implications in medical areas which is generally used to create models for the classification of the attributes that might determine the happening of resulting outcome. The distinctive feature of the Logit is that, the resulting outcome is dichotomous. Generally patients data is being used to develop a proper logistic regression model by identifying the important attributes in the data, which are important in predicting the given outcome. As a result the created model can be used to classify a newly provided patient data through placing in Logit model to calculate the probability $P\left(Y_{i}\right)$ of a given outcome [2].

There are several research studies that use regression models for classification and prediction in biomedical field. In this work [7] authors applied the logistic regression method to predict the probability of fail outcome in Tuberculosis treatment course that might be usable to determine the level of patients' supervision and support. They proved that the developed model-based of Logit achieved $95 \%$ prediction accuracy based on optimal sensitivity and specificity. In another study [8] researchers talk about dichotomous diagnostic test and advantages of using logistic regression models in terms of sensitivity, specificity, 
and likelihood ratios (LRs). The exerted model allows calculating the LRs of diagnostic test results which is conditional on these covariates with an accuracy of $90 \%$. The intended logistic regression approach proves an efficient method to determine the performance of tests at the level of the individual patient risk profile and to examine the effect of patient's characteristics on diagnostic test features. In another study authors applied regression models in the biomechanics field.

\subsection{Metric algorithm K-Nearest Neighbour}

$\mathrm{K}$ nearest neighbour (KNN) is a very simple, the most popular, highly efficient and effective pattern recognition algorithm. KNN is a direct classifier, where a choice is classified based on the class of their nearest neighbour. The marked data is provided to algorithm for training process when training algorithm gets new object, it is assigned to a class that is most common among $\mathrm{k}$ neighbours of the element whose classes are already known.

KNN is also widely used in medicine for various types of tasks. In article [9], authors propose a new algorithm that is based on KNN with a genetic algorithm for detecting heart disease.

In the article [10] considers a combination of decision tree algorithms and the classifier of k-nearest neighbors as an assessment of the selected features for the diagnosis of Parkinson's disease at an early stage. Training data included: speech with several types of sound recordings and Parkinson Handwriting sample datasets used to evaluate the proposed model. In this study authors used one of the approaches to solving the problem of automatic segmentation of discrete speech signal for the diagnosis of disease, in [11] authors consider one of the new approaches to solving this problem. For this purpose, a new type of information functions called TAC-coefficients (throat and acoustic correlation coefficients) is used, which provide sufficient accuracy, efficiency of segmentation for diagnosis of Parkinson's disease.

\subsection{Algorithms based on gradient boosting: XGBoost and LightGBM}

In this study we propose to use algorithms based on the idea of gradient boosting: XGBoost and LightGBM.

XGBoost main features are easy parallelization and impressive prediction accuracy compared to other methods. XGBoost is an efficient and scalable version of the gradient boosting method [12], which has proven itself in several recent machine learning competitions. Authors reported that XGBoost is an ensemble of classification and regression trees that can capture non-linear dependens. The idea of algorithm is to add a classifier in interation and next iteration the classifier has been trained how to improve accuracy base on trained ensemble of trees. In article [13], several machine learning algorithms are used to determine the rule for predicting the diagnosis of influenza by combining clinical signs and symptoms in patients and further assessing the accuracy of the prediction 
model developed using the machine learning algorithm. The XGBoost algorithm showed AUC results: 0.87 on train dataset, 0.79 in test dataset.

LightGBM is a relatively new library [14] and is not widely used in the scientific area [15], but it is widely used in machine learning competitions. The main problem faced by gradient boosting algorithms is that for each function they need to scan all data instances in order to evaluate the gain in information about all possible separation points, which takes a very long time when the dimension of the object is high and the size of the data is large. In the article, the authors proposed a new gradient boosting algorithm, which contains two new techniques: a gradient sampling on one side and an exclusive set of functions for solving a large number of data instances and a large number of functions, respectively. Experimental and theoretical results according have shown that using the LightGBM algorithm can significantly outpace XGBoost in computing speed.

\section{The issue of tuning hyper parameters in models}

One relative disadvantage of these algorithms (XGBoost and LightGBM) is the large number of hyper parameters that are provided to the end user, which in turn can affect the problems of practical use of machine learning algorithms in clinics for diagnosing diseases. Therefore, there is a great attraction for automatic approaches that can optimize the performance of any given learning algorithm for the problem in question. Training time can take considerable time with large amounts of data. To maximize the predictive power of gradient boosting models, you must manually configure the hyper parameters or use automated methods, such as those based on Bayesian optimization.

Bayesian optimization is an effective method for global optimization of objective functions $f: X \rightarrow R$, where $X \subset R^{d}$. Where we decide:

$$
x^{*}=\underset{x \in \mathcal{X}}{\operatorname{argmax}} f(x)
$$

Where $X$ is a compact and convex set. Often, you can access only the perturbed estimates of the function $f()$, which further complicates the optimization. Below we provide a brief description of Bayesian optimization. A more detailed formulation of the Bayes optimization problem is described in [16].

There are two basic steps that must be made when performing Bayesian optimization. The first is to select prior functions that will express assumptions about the function being optimized. To do this, we will pre-select the Gaussian process, due to its flexibility and controllability. Secondly, it is necessary to choose the data collection function that is used to construct the utility function from the posterior model, which allows us to determine the next point to be estimated.

The properties of the Gaussian distribution allow us to calculate predictive averages and variances in closed form [17]. It is determined by the mean function 
$\mu(x)$ and the covariance function $k\left(x, x^{\prime}\right)$. A sample of the Gaussian process is a function, given in the form:

$$
f(x) \sim G P\left(\mu(x), k\left(x, x^{\prime}\right)\right)
$$

where the value of the function at an arbitrary point $\mathrm{x}$ is a random variable distributed according to Gauss. Without any loss in generality, it can be assumed that the previous middle function is a zero function, which makes the Gaussian process a completely definable covariance function. A popular choice for the covariance function is the square exponential function, represented as:

$$
k\left(x, x^{\prime}\right)=\left(-\frac{1}{2 \theta}\left\|x-x^{\prime}\right\|^{2}\right)
$$

where $\theta$ is the length scale parameter. We assume that the length scale is isotropic in our method. Other popular covariance functions include the Mattern kernel, a rational quadratic kernel. In Bayesian optimization, there is the concept of a utility function as a receiving function. The data collection function helps us achieve the optimum of the base function by examining areas where the uncertainty about the function is high and exploring areas where the expected function values can be higher.

Data collection functions can be defined either using criteria based on improvement, or using criteria based on confidence. The upper confidence limit of the predictive GP distribution as a function of data collection. However, paper [16] suggests using a combination of these data collection functions.

The upper limit of the reliability of the Gaussian process:

$$
\alpha_{t}(x)=\mu_{t-1}(x)+\beta_{t}^{\frac{1}{2}} \sigma_{t-1}(x)
$$

where $\beta_{t}=2+2 \cdot d \cdot \log \left(t^{2} \cdot d \cdot b \cdot r \sqrt{\log \left(\frac{4 d a}{\delta}\right)}\right), \sum_{t \geq 1} \pi_{t}^{-1}=1, \pi_{t} \geq 0$

$a, b$ are constants, and $d$ is the dimension of the problem and are given as $a>0, b>0, d>0, r>0, \delta \in(0,1), t \geq 1$. The constants $a, b$ are related to the Lipschitz constant of the objective function $f(x)$.

Now we have discussed the use of a prior over smooth functions using the kernel Mattern, we will focus our attention on computing Bayesian optimization. The role of the data collection function is to find the optimal value. Typically, data collection functions are defined in such a way that high data acquisition corresponds to potentially high values of the target function. Maximization function is used to select the next point at which to evaluate the function. We consider maximizing the probability of improvement $f\left(x^{+}\right)$, where $x^{+}=\operatorname{argmax}_{x \in \mathcal{X}} f\left(x_{i}\right)$. The improvement function is defined as:

$$
I(x)=\max \left\{0, f(x)-f\left(x^{+}\right)\right\}
$$

The new point will be found by maximizing the expected improvement:

$$
x=\underset{x}{\operatorname{argmax}} E\left(\max \left\{0, f(x)_{i+1}-f\left(x^{+}\right)\right\} \mid D_{i}+1\right)
$$


The expected improvement is similar with an analytical solution:

$$
E(I)= \begin{cases}\left(\mu(x)-f\left(x^{+}\right)\right) \Phi(Z)+\sigma(x) \phi(Z), & \text { if } \sigma(x)>0 \\ 0, & \text { if } \sigma(x)=0 .\end{cases}
$$

where $Z=\frac{\mu(x)-f\left(x^{+}\right)}{\sigma(x)} . \Phi($.$) and \phi($.$) are the cumulative distribution and$ probability distribution functions respectively.

Bayesian optimization is a powerful tool for machine learning, where often the problem is not in getting data, but in getting tags. In many ways, this is similar to the usual active learning, but instead of obtaining training data for classification or regression, it allows us to develop structures to effectively solve new types of learning problems. Nevertheless, Bayesian optimization is also a fairly recent addition to machine learning algorithms, and not yet sufficiently studied in user applications.

\section{Materials and methods}

\subsection{Problem description}

The objective of this study is to find better algorithm for machine learning, which will improve the diagnosis of the disease of gastric disorders and provide a result in an acceptable time. In the previous section, we gave a description of the selected algorithms for research on data. We have identified two main points these studies; first one is, used XGBoost and LightGBM gradient boosting algorithms, these algorithms performed well on line data science (Kaggle) competition platforms. LightGBM did not used for similar medical data. LightGBM did not used for similar medical data. Second one is, proving that moderate ML algorithm can outperform by performing several genuine preprocessing on clinical data. The third is, $90 \%$ of the work, which is being done in disease diagnosis, especially in the areas related to health are not open source and being kept secret. The objective is to implement mentioned algorithms from scratch and make them suitable for integration in any clinical decision support system.

\subsection{Description of Data}

In the retrospective analysis study, the medical data related to five digestive systems disorders is considered. The medical data was collected from 1999 to 2014 in the process of routine endoscopic practice for over 1000 subjects, in two hospitals i.e., Samatya and Frunze located in different countries i.e., Turkey and Kyrgyzstan. Patients data selected for those who were confirmed of having mentioned stomach disorders. The disorders prevalence in the dataset is $65 \%$ that is close to WHO statistics.

In the present study, the patient's data has 26 independent attributes of different types described in Table 1 . The attributes are organized into three 
groups; first group shows the patient's history, second group shows symptoms, and last group shows the lab test results. The correlation and significance levels for the attributes are provided Table 1. Prior applying any ML algorithm, the dataset has undergone several normalization and standardization changes. Only those patients data who gave their permission for use had been used in the research in this retrospective study and those patients kept strictly anonymous and confidential.

Table 1: Medical data attributes.

\begin{tabular}{|c|c|c|}
\hline Attributes & Value ranges & Comp.groups \\
\hline \multicolumn{3}{|l|}{ History } \\
\hline Dental probs. & {$[0.01 / 1.00]$} & Comp 1 \\
\hline Sleep disorders & {$[0.01 / 1.00]$} & Comp 1 \\
\hline Constipation & {$[0.01 / 1.00]$} & Comp 1 \\
\hline Age & {$[0$ to 100$]$} & Comp 1 \\
\hline NSAIDs & {$[0.01 / 0.5 / 1.00]$} & Comp 3 \\
\hline Appetite & {$[0.01 / 1.00]$} & Comp 3 \\
\hline Stress & {$[0.01 / 1.00]$} & Comp 4 \\
\hline Gender & {$[0 / 1]$} & Comp 4 \\
\hline Breakfast & {$[0.01 / 1.00]$} & Comp 4 \\
\hline Smoking & {$[0.01 / 1.00]$} & Comp 5 \\
\hline Alcohol & {$[0.01 / 1.00]$} & Comp 5 \\
\hline \multicolumn{3}{|l|}{ Symptoms } \\
\hline Swelling & {$[0.01 / 1.00]$} & Comp 1 \\
\hline Burning & {$[0.01 / 1.00]$} & Comp 1 \\
\hline Souring & {$[0.01 / 1.00]$} & Comp 1 \\
\hline Abdom. pain & {$[0.01 / 1.00]$} & Comp 2 \\
\hline Nausea & {$[0.01 / 1.00]$} & Comp 2 \\
\hline Weakness & {$[0.01 / 1.00]$} & Comp 2 \\
\hline Vomiting & {$[0.01 / 1.00]$} & Comp 2 \\
\hline Diarrhea & {$[0.01 / 1.00]$} & Comp 2 \\
\hline Weightloss & {$[0.01 / 1.00]$} & Comp 3 \\
\hline \multicolumn{3}{|l|}{ Lab tests } \\
\hline Leukocytes (mcL) & {$[4.0 / 10.0]$} & Comp 2 \\
\hline Hemoglobin $(\mathrm{g} / \mathrm{dl})$ & {$[9.0 / 17.0]$} & Comp 3 \\
\hline Stool blood test & {$[0.00 / 1.00]$} & Comp 3 \\
\hline CLO test & {$[0.00 / 1.00]$} & Comp 4 \\
\hline
\end{tabular}

\subsection{Disease groups}

The defined disease groups metrics are shown in Table 3 . The diseases groups are related to five stomach disorders, that are commonly occur in the Middle East and Central Asian populations with a prevalence of $65 \%$. There are two final disease conditions for the ML models in order to perform classification, which 
Table 2: Basic statistics for selected features of dataset.

\begin{tabular}{|l|l|l|l|l|l|l|l|l|}
\hline & Age & HPTEST & WBC & HMG & PLT & SBT & RBC & Stomach diseases \\
\hline count & 1041 & 1041 & 1041 & 1041 & 1041 & 1041 & 1041 & 1041 \\
mean & 33.99 & 0.19 & 6.05 & 12.62 & 160.89 & 0.07 & 4.57 & 1.85 \\
std & 17.40 & 0.39 & 1.56 & 0.90 & 13.58 & 0.25 & 0.41 & 1.99 \\
min & 6 & 0 & 4.2 & 9.8 & 148 & 0 & 3.5 & 0 \\
$25 \%$ & 22 & 0 & 4.8 & 12.2 & 152 & 0 & 4.3 & 0 \\
$50 \%$ & 32 & 0 & 5.4 & 12.5 & 155 & 0 & 4.6 & 1 \\
$75 \%$ & 48 & 0 & 7.3 & 13.2 & 162 & 0 & 4.8 & 4 \\
max & 78 & 1 & 9.8 & 16.2 & 220 & 1 & 5.6 & 6 \\
\hline
\end{tabular}

are 0 representing patients without disorder and 1 representing patients with the defined condition suffering from any stomach disorder. Also several types of disorders were detected in several patients at the same time, these types of diseases were matched into separate classes for subsequent diagnosis of dual diseases in patients. Fig. 1 shows a histogram of the distribution of diseases and such dual diseases as gastritis - reflux and cancer - an ulcer have 16 and 33 patients, respectively.

Table 3: Stomach disorders condition metrics.

\begin{tabular}{|l|l|}
\hline Stomach disorders & Model classification metrics \\
\hline Cancer & {$[0.00 / 1.00]$} \\
Ulcer & {$[0.00 / 1.00]$} \\
Gastritis & {$[0.00 / 1.00]$} \\
Reflux & {$[0.00 / 1.00]$} \\
Flu & {$[0.00 / 1.00]$} \\
Gastritis and Reflux & {$[0.00 / 1.00]$} \\
Cancer and Ulcer & {$[0.00 / 1.00]$} \\
\hline
\end{tabular}

To determine how the various features are related to each other, we constructed two plots: correlation and pair plot, Fig. 2 and 3 respectively. The pair plot allows us to see a distribution of individual variables and relationship between two variables. Paired charts are an excellent method for determining trends for further data analysis. To determine the dependencies between features, 5 features (Age, WBC, HMG, PLT, RBC) have been selected which values are not binary. In Fig. 2 it is possible to separate $5-6$ features that have a correlation above $60 \%$, this gives us the opportunity to reduce the dimension of features in the dataset.

\subsection{Performance measure metrics}

For the comparison of the ML algorithms several performance metrics are used. The selected metrics are briefly described in this section. The experimental re- 


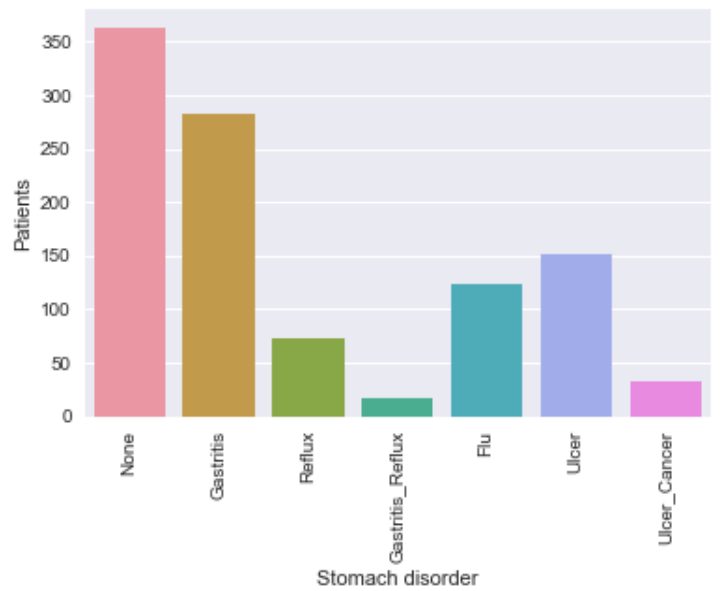

Fig. 1: The histogram of stomach diseases distribution.

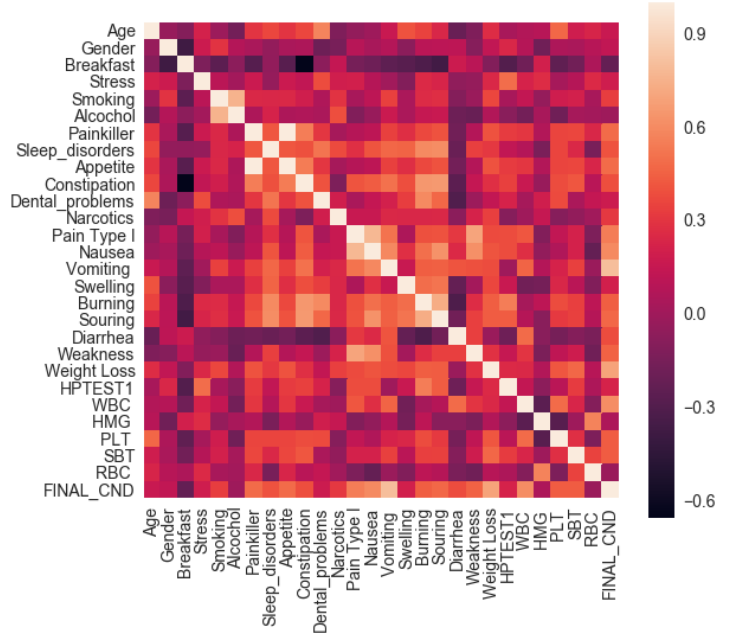

Fig. 2: Correlation matrix between features. 

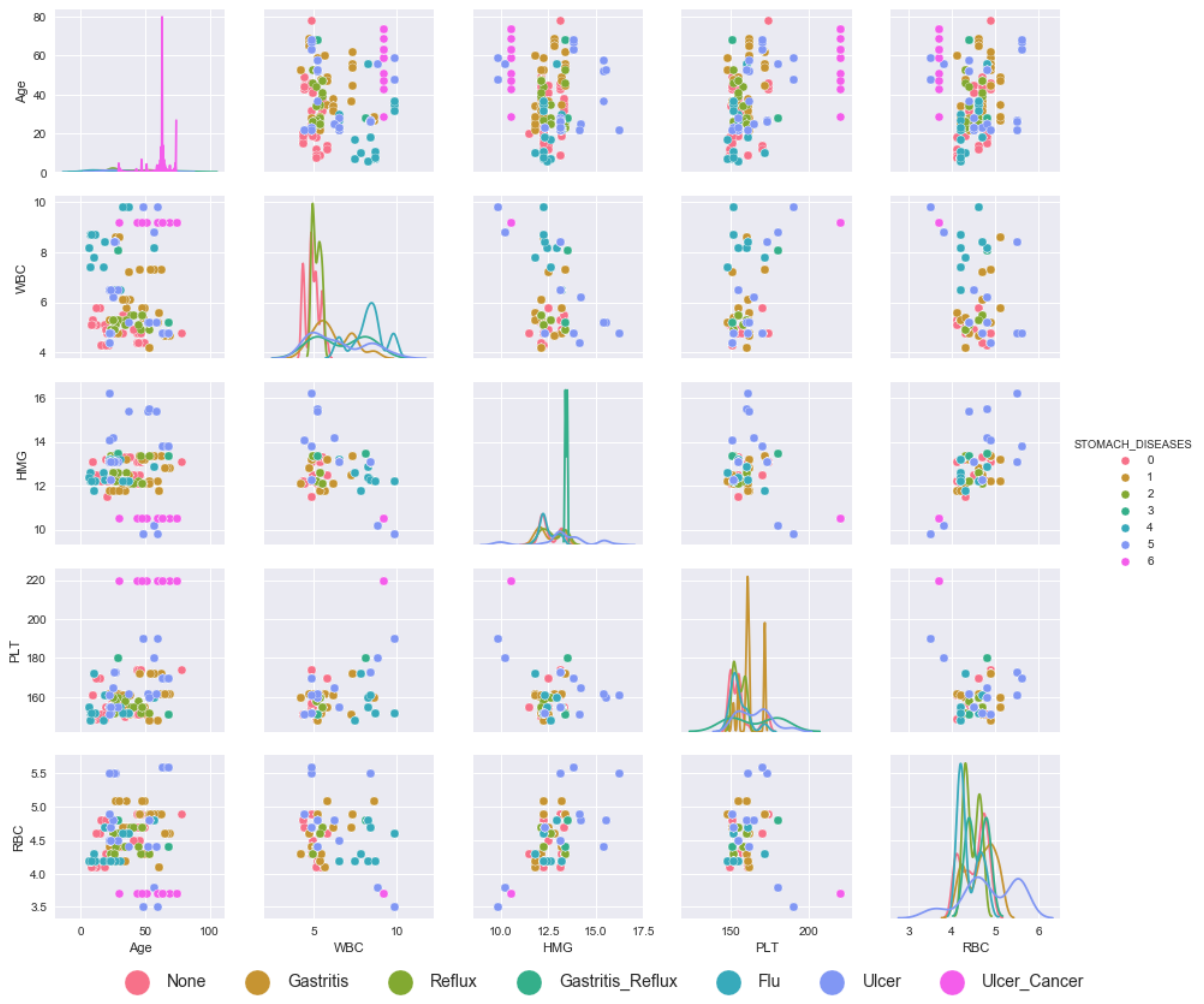

Fig. 3: Dependency between features by classes. 
sults are divided into two groups according to types and provided in Table 4, 5 respectively. Based on these metrics results the ultimate conclusions are made about each algorithm. To estimate accuracy of learning algorithms for selected data, the following metrics were selected: accuracy, precision, recall and f1.

\section{Experimental result and discussions}

In this experimental work, the classification performance of three non-linear ML algorithms are compared and discussed. The medical dataset shown in Table 1, contains patients with five stomach disorders. The prevalence of disease in the dataset is $65 \%$, which is with accordance of WHO reports on gastrointestinal disorders. By using the dataset with such prevalence a robust and an accurate classification model can be created. The resulting models can be integrated into any clinical decision support system which can assist doctors in more precise disease diagnosis.

After the model development the algorithms were given a task to classify patients with stomach disorders. In order to compare the models' performances, we were concentrated on ten metrics shown in Table 4, 5. The underlined metric values indicate which technique is good with respect to which measure.

During the models simulations, the 5 -fold cross validation used, where dataset divided into 5 randomly equal parts, out of which 3 parts used for training and remaining part used to test the model. A learning rate was fixed to 0.3 for assessing the performance of the models

As demonstrated in Table 4, the proposed LightGBM model has the best performance in terms of testing accuracy . The Logit showed a lower performance results as compare to other models. There is no single champion model in classification problems and the best model in terms of accuracy is different from one case to another. Thus we recommend that one should try all alternative models to determine which one will perform best for an underlined data set.

Table 4: Classification performance of models for test dataset.

\begin{tabular}{|l|l|l|l|l|}
\hline & Logit & KNN & XGBoost & LightGBM \\
\hline Accuracy & 0.947 & 0.958 & 0.971 & 0.980 \\
Precision & 0.930 & 0.944 & 0.816 & 0.959 \\
Recall & 0.920 & 0.958 & 0.971 & 0.980 \\
F1 & 0.925 & 0.958 & 0.971 & 0.980 \\
\hline
\end{tabular}

As shown in the Table 5, all models are non-linear but the Logit is more moderate in terms of complexity. The three methods i.e., KNN, XGBoost and LightGBM outperforms Logit according to marginal error making more accurate learning process.

As can be seen from Table 5, the Logit algorithm is still the best result in terms of execution time. LightGBM algorithm suits by two indicators. This fact 
Table 5: Classification performance of models for test dataset.

\begin{tabular}{|l|l|l|l|l|}
\hline & Logit & KNN & XGBoost & LightGBM \\
\hline Runtime (sec) & 0.05 & 0.15 & 0.81 & 0.14 \\
Marginal Error & 0.013 & 0.003 & 0.003 & 0.003 \\
Complexity & Moderate & Complex & Complex & Complex \\
Type & Non-linear & Non-linear & Non-linear & Non-linear \\
\hline
\end{tabular}

requires confirmation of the authors of the article [14], where the author claims that the proposed algorithm shows that the LightGBM shows the best indicators of accuracy and speed of execution.

\section{Conclusion}

In the recent years computer-based disease diagnosis by using machine learning methods have played an important role in improving the quality of medical services. In addition using of computer-based disease diagnosis makes the diagnosis more reliable and therefore increases patient satisfaction. In this study, we developed predictive models using four machine learning algorithms to diagnose stomach disorders. In this study, we studied the capabilities of machine learning algorithms for the diagnosis of diseases of the stomach. The study showed that there is no single method that clearly surpasses all methods in all problem situations. Therefore, one recommendation from this study is to try all alternative models to determine which one will perform best for particular clinical data set. However, the performances varied slightly between models, XGBoost and LightGBM achieved better prediction results (test dataset $97 \%$ and $98 \%$ respectively). One novelty of this paper was LightGBM, which has never been implemented in medical or diagnosis problems. According to results, LoghGBM model tree performed quite competitively with other algorithms. The results of our study suggest that stomach disease can be classified with an accuracy of approximately $94 \%$ with all four machine learning methods. Which is really good in real life problems and we can comfortably suggest that computer-based disease diagnosis can use this four implemented algorithms in their diagnosis problems.

In addition to testing the selected machine learning algorithms on medical data, we highlight a problem of tuning hyper parameters for algorithms. We considered the promising direction of Bayesian optimization for the tuning parameters, but this method also has disadvantages, such as Gaussian processes are not always the best or the simplest solution but even when it is, you need to be very careful when developing a kernel. It can go through a lot of iteration without improvement. These problems are exacerbated by increasing of dataset dimension - more dimensionality means that more samples are required to cover a space, therefore more hyper parameters also need to be tuned.

These studies will continue in this direction - optimization and tuning hyper parameters for machine learning algorithms with a practical bias on big dataset dimensions and data specifics. 


\section{Acknowledgement}

The work was supported by the program-targeted funding projects of the Ministry of Education and Science of Republic of Kazakhstan AP05132648 and BR05236699.

\section{References}

1. Ramesh A. Artificial intelligence in medicine, Annals of The Royal College of Surgeons of England. vol. 86, pp. 334. (2004)

2. Chaudhry B. Systematic review: impact of health information technology on quality, efficiency, and costs of medical care. Annals of internal medicine. vol. 144, pp. 742-752. (2006)

3. WHO, World health statistics 2010, World Health Organization. (2010)

4. Yamada T. Textbook of Gastroenterology, Wiley - Blackwell. (2009)

5. Alkım E. A fast and adaptive automated disease diagnosis method with an innovative neural network model. Neural Networks. 2012. vol 33, pp. 88-96

6. Çomak, E. A decision support system based on support vector machines for diagnosis of the heart valve diseases. Computers in Biology and Medicine. 2007, vol. 37, pp. 21-27.

7. Kalhori S., Nasehi M., Zeng X. J. A logistic regression model to predict high risk patients to fail in tuberculosis treatment course completion. International Journal of Applied Mathematics. vol. 40: pp. 102-107

8. Janssens A., Deng Y., Borsboom G., Eijkemans M., Habbema J., Steyerberg E. A new logistic regression approach for the evaluation of diagnostic test results. Medical decision making. 2005; vol. 25: pp. 168-177

9. Akhiljabbar M., Deekshatulu B. L., Chandrac P. Classification of Heart Disease Using K- Nearest Neighbor and Genetic Algorithm. Procedia Technology. vol. 10, 2013, pp. 85-94.

10. Gupta D., Julka A., Jain S., Aggarwal T., Khanna A., Arunkumar N., de Albuquerque V. H. Optimized cuttlefish algorithm for diagnosis of Parkinson's disease. Cognitive Systems Research. vol. 52, December 2018, pp. 36-48

11. Mussabayev R., Kalimoldayev M., Amirgaliyev Y., Mussabayev T. Automatic speech segmentation using throat-acoustic correlation coefficients. Open Engineering, vol. 6, Issue 1, 2016, pp. 335-346

12. Chen T., Guestrin C. XGBoost: a scalable tree boosting system. Proceedings of the 22nd ACM SIGKDD International Conference on Knowledge Discovery and Data Mining - KDD '16 (2016), pp. 785-794

13. Hung S., Hsieh C., Chen K. Predicting Influenza Infection by Clinical FeatureBased Machine Learning Algorithms. Annals of Emergency Medicine. vol. 72, Issue 4, Supplement, October 2018, pp. S58

14. Ke GL., Meng Q., Finley T., Wang TF., Chen W., Ma WD., Ye QW., Liu TY. LightGBM: A Highly Efficient Gradient Boosting Decision Tree. Advances in Neural Information Processing Systems. (2017)

15. Merembayev T., Yunussov R., Amirgaliyev Y. Machine Learning Algorithms for Stratigraphy Classification on Uranium Deposits. Procedia Computer Science, vol. 150, pp. 46-52, (2019)

16. Brochu E., Cora V. M., de Freitas N. A tutorial on Bayesian optimization of expensive cost functions, with application to active user modeling and hierarchical reinforcement learning. pre-print, 2010. arXiv:1012.2599 
17. Williams C., Rasmussen C. Gaussian processes for machine learning, MIT Press, pp. 4 (2006) 